\title{
Outage performance in relay-assisted overlay cognitive radio networks
}

\begin{abstract}
This paper investigates outage performance under overlay spectrum mode using relayassisted network model. Without harming the performance of the primary user (PU), secondary user ( $\mathrm{SU}$ ) makes use of a relay $(\mathrm{R})$ for transmission as a supportive system besides the direct link transmission between secondary source (S) and secondary destination (D). Outage probability is used to represent the performance parameter for two different cases. In the first case, relay is able to forward the information from source to destination. In the second case, it is assumed the relay suffers an outage and could not decode the information from the source. We consider total probability law in two cases. The outage probability of SU is analyzed in term of different distance between the secondary nodes and model with relay and without relay. The analysis performances are verified by the simulation results.
\end{abstract}

Keyword: Overlay spectrum; Relay-assisted; Decode-and-forward; Outage probability 